\title{
Design and Analysis of Servo Actuator Control System Based on ARM Yang Xin ${ }^{1, a}$, Bo Qingwei ${ }^{2, b}$ and Zhou Changsheng ${ }^{3, c}$ \\ ${ }^{1,2,3}$ School of Mechanical Engineering, Nanjing University of Science and Technology, Jiangsu Nanjing 210094, China \\ ayangxin3935@126.com, b bergqingwei@sina.com, chenxiong@njust.edu.cn
}

Key words: LPC2292; Servo system; PID; Position control

\begin{abstract}
A servo control system and servo position signal acquisition system based on PHILIPS ARM7 series kernel LPC2292 were designed. The principle and construction of the position control module was discussed in detail. The accuracy of controlling system was verified using Matlab code. Results show that the system can perform accurate position control of four sets of digital servo and make the rudder missiles of guided rockets realize position control more conveniently and accurately.
\end{abstract}

\section{Introduction}

With the development of society, the servo system is more and more widely used in various industrial fields such as precision equipment and sophisticated institutions. In recent years, the national aerospace industry, precise guidance weapons and a variety of rare earth motor are developing continuously. The request of overall performance of the servo control system is becoming higher and higher ${ }^{[1-3]}$.As the executive body of the missile's control system, servo system is to convert the control command to the corresponding deflection of thrust direction, thereby producing the desired yaw moment to change the direction of flight, changing the direction of flight or overcoming the interference of steady flight, so its performance largely affects the guidance precision of missiles and determines the dynamic performance of the missile flight. It is a typical position servo system.

This paper based on ARM7 microcontroller system, build the steering servo control system with 32-bit ARM7 LPC2292 kernel chip as the core processor, using ARM microcontroller to perform accurate position control of four sets of digital servo, gather feedback signal and do data calculation and analysis with Matlab platform, to eventually realize the ultra precise control of servo's position.

\section{The hardware structure of the control system design}

This paper aims to design the hardware and software based on ARM7 microcontroller system ${ }^{[2]}$. The main hardware circuit includes core chipLPC2292 produced by PHILIPS company, digital servo steering gear DS - $3120 \mathrm{mg}$, PWM generation and receiving module, power module, AD sampling module, SR232 interface circuit composition and so on.

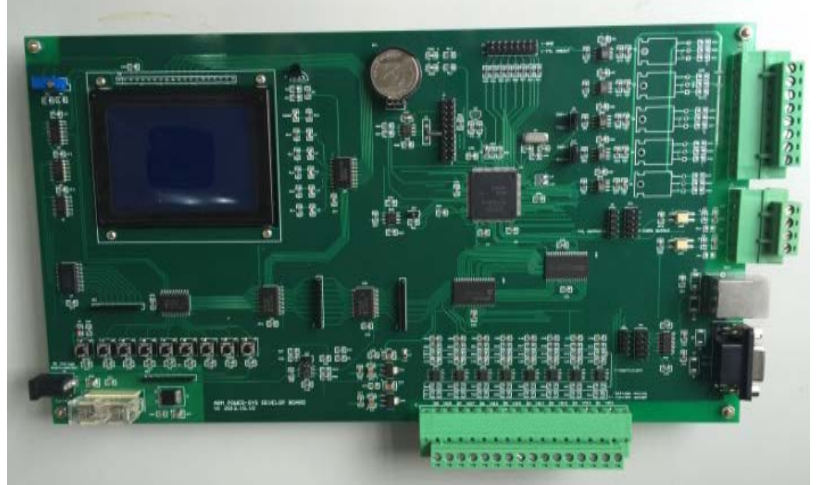

Figure 1. ARM development board hardware diagram 


\subsection{The application of kernel chip LPC2292 and the PWM module in the system}

The LPC2292 has seven match registers, allowing up to six single edge controlled or three double edge controlled PWM outputs, or a mix of both types. The PWM function is an additional feature, based on match register events. Block diagram of PWM is shown in Figure.2. The parts added to standard Timer block are on the right and top of the figure.

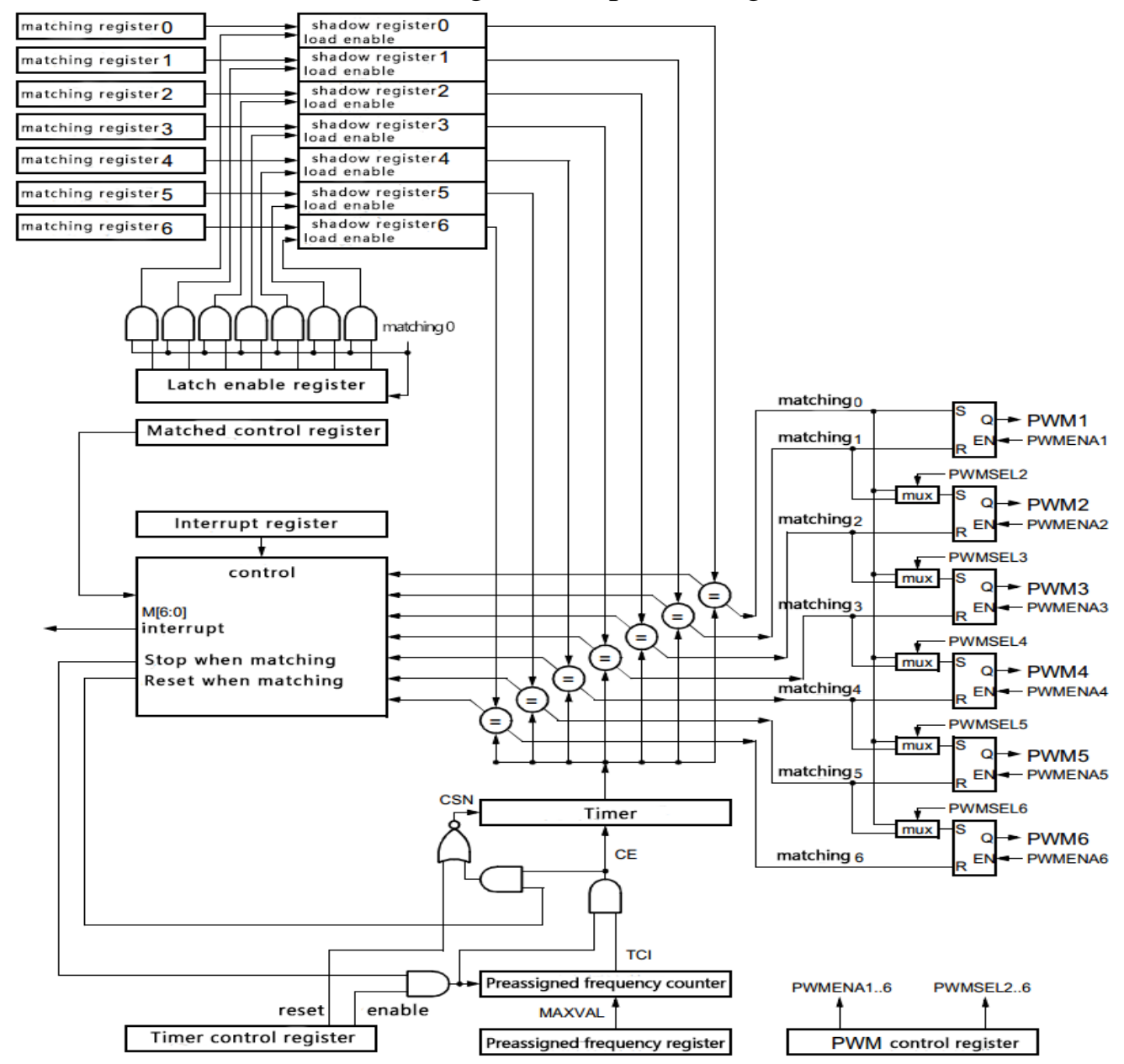

Figure 2.Block diagram of PWM

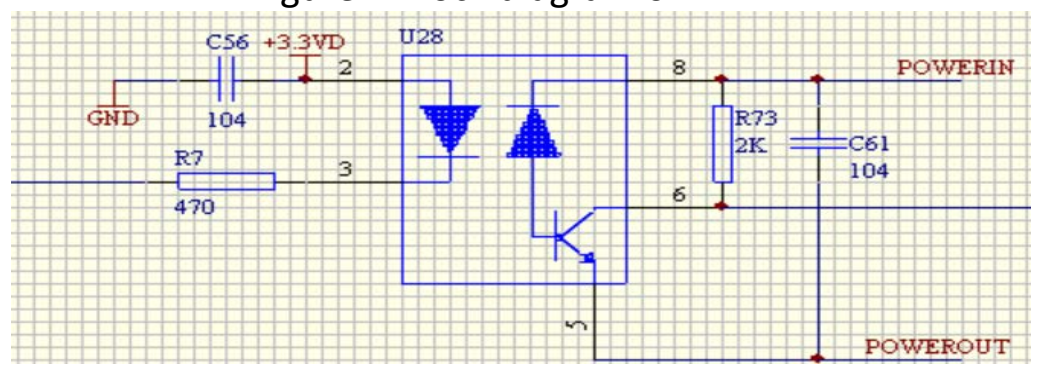

Figure 3.Hardware schematic diagram of PWM modules

\subsection{Applications of AD sampling modules and power modules in this system}

The diagram in Figure.4 is the module of A/D sampling ${ }^{[3]}$. ARM does AD sampling on output current and then modules the output PWM duty cycle with current regulator after comparing with current reference value.

But the output current of power produces ripples due to the modulation of PWM, thus direct sampling will affect the control effect on ARM. To eliminate the influence of the ripples on current control, AD sampling current value must be conducted with average digital filtering in ARM. Simulation modules take into account the effects of random noise on the AD sampling, and transform the sampling results into register values as AD results stored in ARM. 


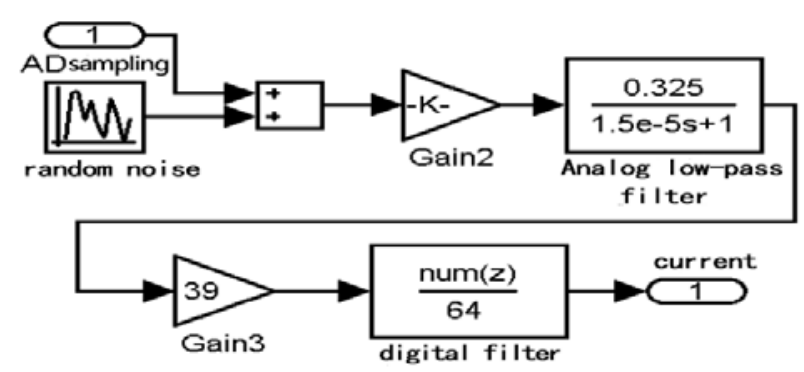

Figure 4.ADsampling module

\section{Structures and principles of servo controller systems}

PID control algorithm is widely used in modern industrial control. The algorithm is simple and practical, but for complex nonlinear time-varying system it is not well controlled with high accuracy. Therefore intelligent control algorithm is proposed to optimize the classical PID, meeting the requirements of the control system. Formula 2.1 below shows the principle of the controller. The mathematical model of PID controller:

$$
u(t)=k_{P}\left[e(t)+\frac{1}{T_{I}} \int_{0}^{t} e(t) d t+\frac{T_{D} d e(t)}{d t}\right]
$$

In the formula, $e(t)=r(t)-c(t)$ is the deviation of the expected value $r(t)$ and the actual value $c(t)$. The deviation is the core of PID control algorithm: adjustment based on the presence of bias. According to the expression above, it can be seen that the control algorithm has three parameters that is proportion $k_{P}$, Integration time $T_{I}$ and Derivative time $T_{D}$.

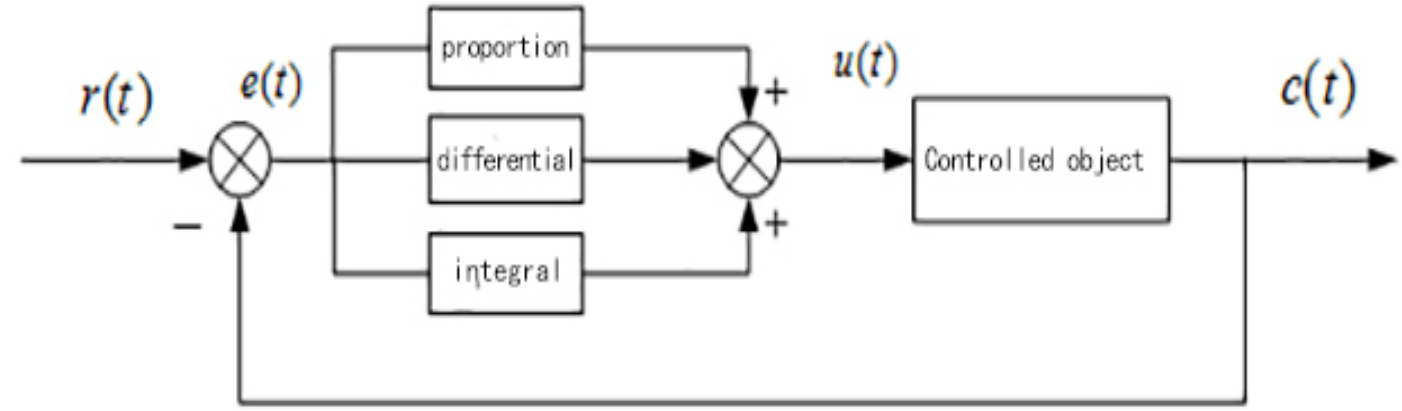

Figure 5. PID Schematic diagram

Practical applications are using the digital signal, thus the above equation is discretized.

$$
\left\{\begin{array}{l}
t \approx k T \quad(k=0,1,2, \ldots) \\
\int_{0}^{t} e(t) d t \approx T \sum_{i=0}^{k} e(i T)=T \sum_{i=0}^{k} e(i) \\
\frac{d e(t)}{d t} \approx \frac{e(k T)-e((k-1) T)}{T}=\frac{e(k)-e(k-1)}{T}
\end{array}\right.
$$

Process to get the PID Discretization of the following expressions:

$$
u(k)=k_{P}\left[e(k)+\frac{T}{T_{I}} \sum_{i=0}^{k} e(i)+T_{D} \frac{e(k)-e(k-1)}{T}\right]
$$

In the formula, $T$ is the sampling period. This is a position-type expression. In some systems, incremental expressions are required. The following formula can be obtained according to the last: 


$$
u(k-1)=k_{P}\left[e(k-1)+\frac{T}{T_{I}} \sum_{i=0}^{k-1} e(i)+T_{D} \frac{e(k-1)-e(k-2)}{T}\right]
$$

Two subtraction can get incremental PID expression:

$$
\Delta u(k)=k_{P}\left[\Delta e(k)+\frac{T}{T_{I}} e(k)+T_{D} \frac{\Delta e(k)-\Delta e(k-1)}{T}\right]
$$

Among it, $\Delta e(k)=e(k)-e(k-1)$.

To compare position with incremental PID expression, we can see the demand to accumulate all positional deviation, while incremental deals with three times as long as the deviation, thus the computation is relatively small. At the same time, positional PID control direct output, but incremental PID is not, so the latter can obtain a more stable control effect. This paper adopts the incremental PID control method for motor control.

\section{Feedback signal acquisition and analysis of Matlab}

The control system hardware platform is built upon request to achieve the accurate collection of four sets of feedback signal for the Servo. PCI data acquisition card is used for the main components of the data acquisition model. The corresponding data acquisition system is established via connecting the card with PC and ARM microcontrollers ${ }^{[4]}$. Several experiments in signal acquisition will be done to achieve four servo feedback signal acquisition and recording at the same time.

Fuzzy neural network PID is capable of studying on line and adjusting the weight coefficient quickly by the error response propagation. The setting process of PID parameters of fuzzy neural network is shown in Figure 6.
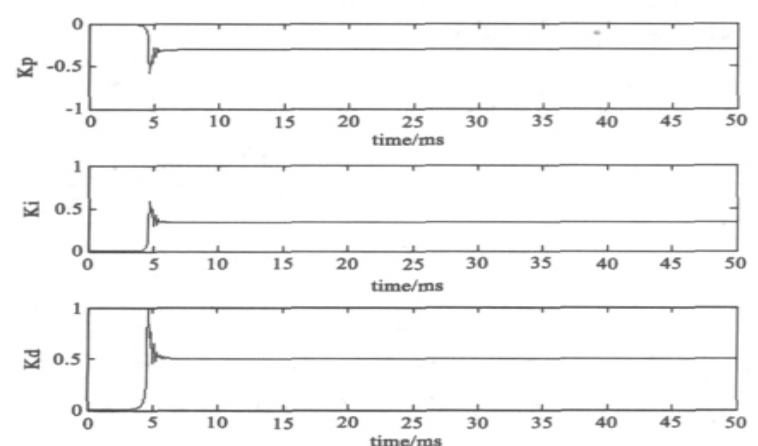

Figure 6.The setting process of PID parameters of fuzzy neural networks

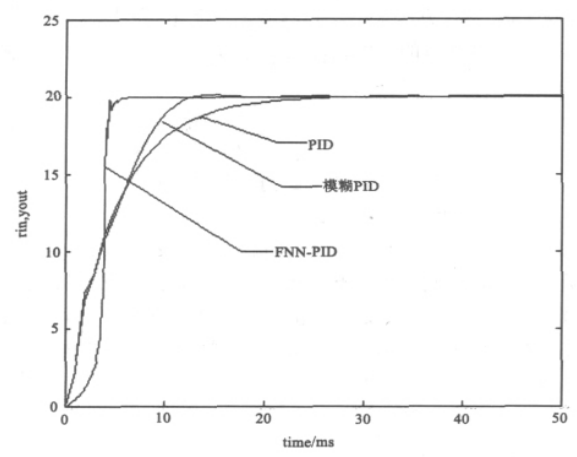

Figure 7. Comparison of the step response of each controller

In the simulation software MATLAB, the steering gear mathematical model is discrete, and the above improved algorithm is used to control the simulation test ${ }^{[5]}$. The system is given a 20 degree position deflection signal, then the simulation process is comparing PID controller and fuzzy PID ${ }^{\text {[6] }}$ controller with the fuzzy neural network PID controller designed in this paper, as shown in Figure7.

\section{Conclusion}

This article is able to achieve accurate positioning of servo motor based on LPC2292 and servo system position control method. Servo position information can be accurately collected by the data acquisition card. The information is imported through improved PID control algorithm after AD conversion. This algorithm is employed to adjust servo position, and ultimately the system achieves close-loop control. The design verifies that servo motor position control system has advantages such as strong anti-magnetic interference, accurate positioning, etc. Therefore, the system has been widely used in missile rudder. 


\section{References}

[1]Caitlin H. Raytheon Continue Sidewinder Development[J]. Jane’s Missiles \& Rockets, 1(2010), 56-57.

[2]Doug R. Silent Eagle fires AMRAAM from a conformal weapons bay[J]. Jane's Missiles \& Rockets, 9(2010), 3.

[3]Williams K, BrownD. Electrically powered actuator design. NASA/USAF/Navy, 5(1997), 34-37.

[4]G.MadhusudhanaRao, B.V.SankerRamB.Smapath Kumar, K.VijayKumar. Speed Control of BLDC Motor Using DSP. International Journal of Engineering Science and Technology, 2 (3) 2010, 143.

[5]Nicholas Moehle, Stephen Boyd. Optimal current waveforms for brushless permanent magnet motors. International Journal of Control, 88(7), 2015, 1389-1399.

[6]Wu Yingzhe, Liu Hui, Bi Ye. Study on Missile Rudder Servo System Based on Mixed Fuzzy-PID Control Algorithm[C], IEEE International Conference on Mechatronics and Automation. Beijing, China, 3(2011), 2072-2077. 